\title{
The Homotopy Types of $S U(3)$-gauge Groups over Simply Connected 4-manifolds
}

\author{
by
}

\author{
Stephen TheriaulT
}

\begin{abstract}
We classify the integral homotopy types of gauge groups of principal $S U(3)$-bundles over a simply-connected Spin 4-manifold $M$. When $M$ is non-Spin, a similar classification holds once localization is allowed.
\end{abstract}

2010 Mathematics Subject Classification: Primary 54P15; Secondary 54C35.

Keywords: gauge group, homotopy type, 4-manifold.

\section{$\S 1$. Introduction}

Let $M$ be a simply-connected closed 4-manifold and let $G$ be a simple, simplyconnected compact Lie group. Let $P \rightarrow M$ be a principal $G$-bundle. The gauge group of this bundle is the group of $G$-equivariant automorphisms of $P$ which fix $M$. As $[M, B G]=\mathbb{Z}$, the principal $G$-bundles over $M$ are classified by the second Chern class. If $c_{2}(P)=k$, let $\mathcal{G}_{k}(M, G)$ be the gauge group of $P$. In [CS] it was shown that for a fixed $M$ and $G$, the gauge groups $\left\{\mathcal{G}_{k}(M, G) \mid k \in \mathbb{Z}\right\}$ have only finitely many distinct homotopy types. In recent years there has been considerable interest in determining the precise number of homotopy types in cases related to the $U(1) \times S U(2) \times S U(3)$-gauge groups associated to the standard model in physics. For example, there are six homotopy types of $S U(2)$-gauge groups over $S^{4}[\mathrm{~K}]$; six homotopy types of $S U(2)$-gauge groups over a Spin 4-manifold and four homotopy types of $S U(2)$-gauge groups over a non-Spin 4-manifold [KT]; and eight homotopy types of $S U(3)$-gauge groups over $S^{4}$ [HK2]. The purpose of this paper is to determine the number of homotopy types of $S U(3)$-gauge groups over Spin and non-Spin 4-manifolds.

Communicated by T. Ohtsuki. Received May 4, 2011. Revised September 22, 2011, and October 12, 2011.

S. Theriault: Institute of Mathematics, University of Aberdeen,

Aberdeen AB24 3UE, United Kingdom:

e-mail: s.theriault@abdn.ac.uk 
To simplify notation, let $\mathcal{G}_{k}(M)=\mathcal{G}_{k}(M, S U(3))$. If $a, b$ are two integers, let $(a, b)$ be the greatest common divisor of $|a|$ and $|b|$.

Theorem 1.1. Let $M$ be a simply-connected closed 4-manifold. The following hold:

(a) if $M$ is a Spin manifold then there is an integral homotopy equivalence $\mathcal{G}_{k}(M)$ $\simeq \mathcal{G}_{k^{\prime}}(M)$ if and only if $(24, k)=\left(24, k^{\prime}\right)$

(b) if $M$ is a non-Spin manifold then an integral homotopy equivalence $\mathcal{G}_{k}(M) \simeq$ $\mathcal{G}_{k^{\prime}}(M)$ implies that $(12, k)=\left(12, k^{\prime}\right)$, while $(12, k)=\left(12, k^{\prime}\right)$ implies that there is a homotopy equivalence $\mathcal{G}_{k}(M) \simeq \mathcal{G}_{k^{\prime}}(M)$ after localizing rationally or at any prime.

Theorem 1.1 implies that there are eight distinct integral homotopy types for $\mathcal{G}_{k}(M)$ when $M$ is Spin. It also says there are at least six integral homotopy types for $\mathcal{G}_{k}(M)$ when $M$ is non-Spin. One would obtain exactly six homotopy types if the localized statement in part (b) could be replaced by an integral one. One approach for doing this is to try to stitch together the localized results using Sullivan's arithmetic square, but this appears to be technically difficult. A more direct approach seems to be beyond current methods.

The proof of Theorem 1.1 depends on both general methods - such as decompositions in homotopy theory and a lemma for estimating an upper bound on the number of distinct gauge groups - and on information specific to both $S U(3)$ and the 4-manifold $M$ (to distinguish the Spin and non-Spin cases). This approach should be applicable to other cases.

The author would like to thank the referee for a careful reading of the paper and many constructive comments.

\section{$\S 2$. Preliminary homotopy theory}

The results in this section are kept somewhat general, keeping in mind the more general statements to come in Section 4 . Let $M$ be a simply-connected, closed 4-manifold and let $G$ be a simple, simply-connected, compact Lie group. For a topological group $H$, let $B H$ be its classifying space. Let $P \rightarrow M$ be a principal $G$-bundle, whose second Chern class is the integer $k$. Let $\operatorname{Map}_{k}(M, B G)$ be the component of the space of continuous unbased maps from $M$ to $B G$ which contains the map inducing $P$. Similarly, let $\operatorname{Map}_{k}^{*}(M, B G)$ be the space of continuous pointed maps from $M$ to $B G$ which contains the map inducing $P$. Observe that there is a fibration $\operatorname{Map}_{k}^{*}(M, B G) \rightarrow \operatorname{Map}_{k}(M, B G) \stackrel{\text { ev }}{\longrightarrow} B G$, where ev evaluates a map at the basepoint of $M$. By $[\mathrm{AB}]$, there is a homotopy equivalence 
$B \mathcal{G}_{k}(M, G) \simeq \operatorname{Map}_{k}(M, B G)$. The evaluation fibration therefore determines a homotopy fibration sequence

$$
G \stackrel{\partial_{k}^{M}}{\longrightarrow} \operatorname{Map}_{k}^{*}(M, B G) \rightarrow B \mathcal{G}_{k}(M, G) \stackrel{\text { ev }}{\longrightarrow} B G
$$

which defines the map $\partial_{k}^{M}$. In the special case when $M=S^{4}$, it is well known that $\operatorname{Map}_{k}^{*}\left(S^{4}, B G\right) \simeq \operatorname{Map}_{0}^{*}\left(S^{4}, B G\right)$ for every $k$. It is usual to write $\Omega_{0}^{3} G$ for $\operatorname{Map}_{0}^{*}\left(S^{4}, B G\right)$. As well, to distinguish the gauge group for this case, let $\mathcal{G}_{k}=$ $\mathcal{G}_{k}\left(S^{4}\right)$.

Since $M$ is a simply-connected, closed 4-manifold, Poincaré duality implies that $M$ has one zero cell, $d$ two-cells for some nonnegative integer $d$, and one four-cell. Thus there is a homotopy cofibration sequence

$$
S^{3} \stackrel{f}{\rightarrow} \bigvee_{i=1}^{d} S^{2} \rightarrow M \stackrel{q}{\rightarrow} S^{4} \stackrel{\Sigma f}{\rightarrow} \bigvee_{i=1}^{d} S^{3}
$$

where $f$ is the attaching map for the top cell of $M$ and $q$ is the pinch map to the top cell. Since $\operatorname{Map}_{k}(, B G)$ and $\operatorname{Map}_{k}^{*}(, B G)$ are functors, when applied to the pinch map $M \stackrel{q}{\rightarrow} S^{4}$ we obtain a homotopy fibration diagram

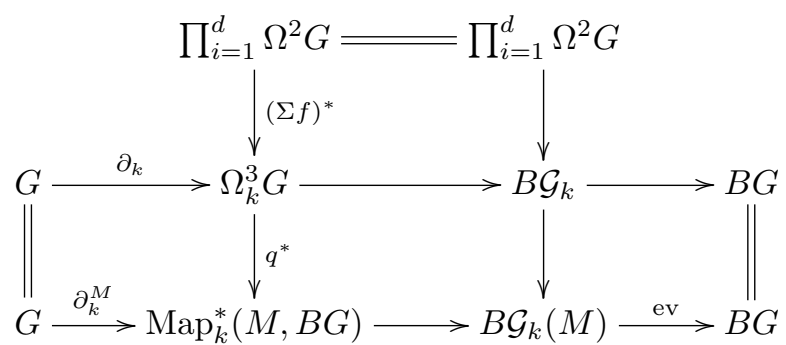

In particular, $\partial_{k}^{M}$ factors through $\partial_{k}$.

The map $\partial_{k}$ is well understood. Let $i: S^{3} \rightarrow G$ be the inclusion of the bottom cell and let $1: G \rightarrow G$ be the identity map. The following lemma proved in [L] determines the homotopy class of the triple adjoint of $\partial_{k}$.

Lemma 2.1. The adjoint of the map $G \stackrel{\partial_{k}}{\longrightarrow} \Omega_{0}^{3} G$ is homotopic to the Samelson product $S^{3} \wedge G \stackrel{\langle k i, 1\rangle}{\longrightarrow} G$.

The linearity of the Samelson product implies that $\langle k i, 1\rangle \simeq k\langle i, 1\rangle$. Adjointing therefore implies the following.

Corollary 2.2. There is a homotopy $\partial_{k} \simeq k \circ \partial_{1}$. 
§3. Decompositions of gauge groups and one case of Theorem 1.1

One direction in Theorem 1.1(a) is an easy consequence of a decomposition for $\mathcal{G}_{k}(M)$ when $M$ is a Spin manifold, together with the classification of the homotopy types of $\mathcal{G}_{k}$ in [HK2].

The fact that $M$ is a Spin manifold is equivalent to the fact that in the homotopy cofibration $S^{3} \stackrel{f}{\rightarrow} \bigvee_{i=1}^{d} S^{2} \rightarrow M$ defining $M$, the map $f$ has the property that $\Sigma f$ is null homotopic. If $M$ is not a Spin manifold then $\Sigma f$ is essential. On the other hand, since $\pi_{4}\left(\bigvee_{i=1}^{d} S^{3}\right) \cong \bigoplus_{i=1}^{d} \mathbb{Z} / 2 \mathbb{Z}$, we have $\Sigma f$ null homotopic after localizing away from 2 . In either case, the induced map $(\Sigma f)^{*}$ in $(1)$ is null homotopic. This was used in [Th1] to prove the following.

Theorem 3.1. Let $M$ be a simply-connected closed 4-manifold. The following hold:

(a) if $M$ is a Spin manifold then there is an integral homotopy equivalence

$$
\mathcal{G}_{k}(M, G) \simeq \mathcal{G}_{k}(G) \times \prod_{i=1}^{d} \Omega^{2} G
$$

(b) if $M$ is not a Spin manifold then there is a homotopy equivalence

$$
\mathcal{G}_{k}(M, G) \simeq \mathcal{G}_{k}(G) \times \prod_{i=1}^{d} \Omega^{2} G
$$

after localizing away from 2.

Corollary 3.2. Let $M$ be a simply-connected Spin 4-manifold. If $(24, k)=\left(24, k^{\prime}\right)$ then $\mathcal{G}_{k}(M) \simeq \mathcal{G}_{k^{\prime}}(M)$.

Proof. Since $(24, k)=\left(24, k^{\prime}\right)$, by [HK2] there is an integral homotopy equivalence $\mathcal{G}_{k} \simeq \mathcal{G}_{k^{\prime}}$. Since $M$ is a Spin manifold, Theorem 3.1 states that there is an integral homotopy equivalence $\mathcal{G}_{k}(M) \simeq \mathcal{G}_{k} \times \prod_{i=1}^{d} \Omega^{2} S U(3)$. Thus we obtain a string of integral homotopy equivalences

$$
\mathcal{G}_{k}(M) \simeq \mathcal{G}_{k} \times \prod_{i=1}^{d} \Omega^{2} S U(3) \simeq \mathcal{G}_{k^{\prime}} \times \prod_{i=1}^{d} \Omega^{2} S U(3) \simeq \mathcal{G}_{k^{\prime}}(M)
$$

When $M$ is not Spin, the decomposition in Theorem 3.1(b) gives no 2-primary information. So in this case different methods are needed to distinguish homotopy types. 


\section{$\S 4$. A counting lemma}

The factorization of $\partial_{k}^{M}$ through $\partial_{k}$ in (1) implies that there is a homotopy pullback diagram

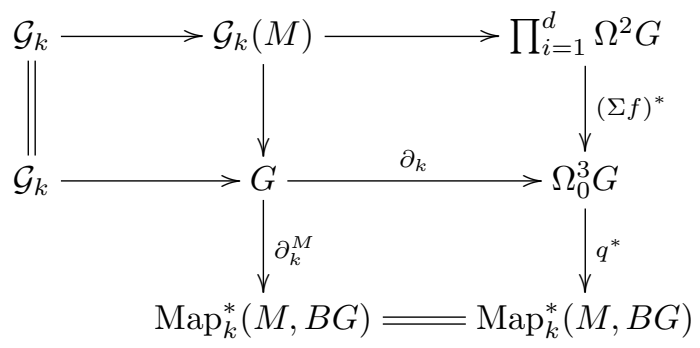

In determining the homotopy types of the gauge groups $\mathcal{G}_{k}$ an important intermediate step is to determine the order of the map $\partial_{k}$. In trying to do the same for the gauge groups $\mathcal{G}_{k}(M)$ we run into a problem: $\operatorname{Map}_{k}^{*}(M, B G)$ need not be an $H$-space so the set of homotopy classes $\left[G, \operatorname{Map}_{k}^{*}(M, B G)\right]$ need not have a group structure. Therefore it makes no sense to speak of the order of $\partial_{k}^{M}$. What follows is a means of sidestepping this problem.

To motivate the statement in Lemma 4.2, it may be useful to recall a special case of [Th2, Lemma 3.1], which deals with the case of $\mathcal{G}_{k}$.

Lemma 4.1. Suppose the map $G \stackrel{\partial_{1}}{\longrightarrow} \Omega_{0}^{3} G$ has order $m$, where $m$ is finite. If $(m, k)=\left(m, k^{\prime}\right)$ then $\mathcal{G}_{k}$ and $\mathcal{G}_{k^{\prime}}$ are homotopy equivalent when localized rationally or at any prime.

Modifying to the case of $\mathcal{G}_{k}(M)$, we prove the following.

Lemma 4.2. Suppose the map $G \stackrel{\partial_{1}}{\longrightarrow} \Omega_{0}^{3} G$ has order $m$, where $m$ is finite. If $(m, k)=\left(m, k^{\prime}\right)$ then $\mathcal{G}_{k}(M)$ and $\mathcal{G}_{k^{\prime}}(M)$ are homotopy equivalent when localized rationally or at any prime.

Proof. Since $\partial_{1}$ has order $m$, the homotopy class of $\partial_{1}$ generates a cyclic subgroup $S=\mathbb{Z} / m \mathbb{Z}$ in $\left[G, \Omega_{0}^{3} G\right]$. Suppose $(m, k)=\left(m, k^{\prime}\right)=l$. Then $k=l t$ and $k^{\prime}=l t^{\prime}$ for integers $t$ and $t^{\prime}$ which are units in $\mathbb{Z} / m \mathbb{Z}$. Let $s$ and $s^{\prime}$ be integers such that $s t \equiv 1(\bmod m)$ and $s^{\prime} t^{\prime} \equiv 1(\bmod m)$. Observe that $k s \equiv l(\bmod m)$ and $k^{\prime} s^{\prime} \equiv l(\bmod m)$. Thus the composites $G \stackrel{\partial_{1}}{\longrightarrow} \Omega_{0}^{3} G \stackrel{k s}{\longrightarrow} \Omega_{0}^{3} G$ and $G \stackrel{\partial_{1}}{\longrightarrow} \Omega_{0}^{3} G \stackrel{k^{\prime} s^{\prime}}{\longrightarrow}$ $\Omega_{0}^{3} G$ both represent the homotopy class [l] in $S$. That is, $k s \circ \partial_{1}$ is homotopic to $k^{\prime} s^{\prime} \circ \partial_{1}$. This together with Lemma 2.1 implies that there is a string of homotopies $\partial_{k s}^{M}=q^{*} \circ \partial_{k s} \simeq q^{*} \circ k s \circ \partial_{1} \simeq q^{*} \circ k^{\prime} s^{\prime} \circ \partial_{1} \simeq q^{*} \circ \partial_{k^{\prime} s^{\prime}}=\partial_{k^{\prime} s^{\prime}}^{M}$. Consequently, $\mathcal{G}_{k s}(M) \simeq \mathcal{G}_{k^{\prime} s^{\prime}}(M)$. Note that this holds integrally. 
Now fix a prime $p$ and localize at $p$. There are two cases. First, suppose that $(m, p)=1$. Then $m$ and $p$ have no common factors so $m$ is a unit mod- $p$. Thus the power map $\Omega_{0}^{3} G \stackrel{m}{\longrightarrow} \Omega_{0}^{3} G$ is a homotopy equivalence, implying that $\partial_{1}$ has order 1. That is, $\partial_{1}$ is null homotopic. So by Lemma 2.1, $\partial_{k} \simeq k \circ \partial_{1}$ is null homotopic for any integer $k$, and therefore $\bar{\partial}_{k} \simeq q^{*} \circ \partial_{k}$ is null homotopic. Thus $\mathcal{G}_{k}(M) \simeq G \times \Omega_{0}^{4} G$. As this is true for any integer $k$, we have $\mathcal{G}_{k}(M) \simeq \mathcal{G}_{k^{\prime}}(M)$ for any integers $k$ and $k^{\prime}$. Second, suppose that $(m, p)=p$. Since $s$ is a unit in $\mathbb{Z} / m \mathbb{Z}$, we have $(m, s)=1$. Therefore $(s, p)=1$, which implies that $s$ is a unit mod- $p$. Thus the power map $\Omega^{3} G_{0} \stackrel{s}{\rightarrow} \Omega_{0}^{3} G$ is a homotopy equivalence. This implies that the sequence $\prod_{i=1}^{d} \Omega^{2} G \stackrel{(\Sigma f)^{*}}{\longrightarrow} \Omega_{0}^{3} G \stackrel{q^{*} \circ s}{\longrightarrow} \operatorname{Map}_{k}^{*}(M, B G)$ is a fibration up to homotopy. Now consider the homotopy pullback diagram

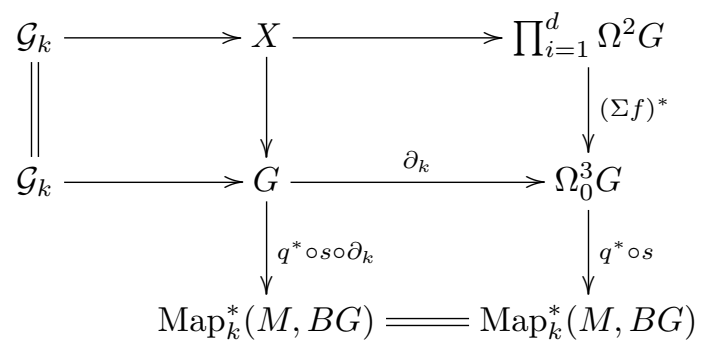

which defines the space $X$. On the one hand, by (2) we have $X \simeq \mathcal{G}_{k}(M)$. On the other hand, by Lemma 2.1, $\partial_{k s} \simeq s \circ \partial_{k}$, and by (1), $\partial_{k s}^{M} \simeq q^{*} \circ \partial_{k s}$. Thus there is a string of homotopies $q^{*} \circ s \circ \partial_{k} \simeq q^{*} \circ \partial_{k s} \simeq \partial_{k s}^{M}$. Therefore $X$ is also homotopy equivalent to the homotopy fibre of $\partial_{k s}^{M}$, which is $\mathcal{G}_{k s}(M)$. Hence $\mathcal{G}_{k}(M) \simeq \mathcal{G}_{k s}(M)$. Similarly, $\mathcal{G}_{k^{\prime}}(M) \simeq \mathcal{G}_{k^{\prime} s^{\prime}}(M)$. Consequently, there is a string of homotopy equivalences $\mathcal{G}_{k}(M) \simeq \mathcal{G}_{k s}(M) \simeq \mathcal{G}_{k^{\prime} s^{\prime}}(M) \simeq \mathcal{G}_{k^{\prime}}(M)$.

Finally, consider the rational case. Since $m$ is a unit in $\mathbb{Q}$, arguing as in the first case above shows that $\mathcal{G}_{k}(M) \simeq G \times \Omega_{0}^{4} G \simeq \mathcal{G}_{k^{\prime}}(M)$ for any integers $k$ and $k^{\prime}$.

\section{$\S 5$. Some preliminary calculations}

In this section we collect some calculations of groups of homotopy classes of maps which will be used later. We begin by stating some of Mimura and Toda's results $[\mathrm{MT}]$ on the homotopy groups of $S U(3)$. Recall that there is a canonical fibration $S^{3} \stackrel{i}{\rightarrow} S U(3) \stackrel{\pi}{\rightarrow} S^{5}$. As usual, the stable class in $\pi_{n+1}\left(S^{n}\right) \cong \mathbb{Z} / 2 \mathbb{Z}$ is labelled $\eta$ and the stable 3 -primary class in $\pi_{n+3}\left(S^{n}\right)$ is labelled $\alpha$.

Lemma 5.1. The homotopy groups $\pi_{i}(S U(3))$ for $4 \leq i \leq 11$ are as follows:

\begin{tabular}{|c|c|c|c|c|c|c|c|c|}
\hline$i$ & 4 & 5 & 6 & 7 & 8 & 9 & 10 & 11 \\
\hline$\pi_{i}$ & 0 & $\mathbb{Z}$ & $\mathbb{Z} / 6 \mathbb{Z}$ & 0 & $\mathbb{Z} / 12 \mathbb{Z}$ & $\mathbb{Z} / 3 \mathbb{Z}$ & $\mathbb{Z} / 30 \mathbb{Z}$ & $\mathbb{Z} / 4 \mathbb{Z}$ \\
\hline
\end{tabular}


Further, the following hold:

(a) the generator of $\pi_{5}(S U(3))$ is represented by a map c: $S^{5} \rightarrow S U(3)$ with the property that the composite $S^{5} \stackrel{c}{\rightarrow} S U(3) \stackrel{\pi}{\rightarrow} S^{5}$ has degree 2 ;

(b) a representative of the generator of the 2-component of $\pi_{6}(S U(3))$ is given by the composite $S^{6} \stackrel{\eta}{\rightarrow} S^{5} \stackrel{c}{\rightarrow} S U(3) ;$

(c) a different representative of the generator of the 2-component of $\pi_{6}(S U(3))$ is given by the composite $S^{6} \stackrel{\nu^{\prime}}{\rightarrow} S^{3} \stackrel{i}{\rightarrow} S U(3)$;

(d) a representative of the generator of the 3-component of $\pi_{6}(S U(3))$ is given by the composite $S^{6} \stackrel{\alpha}{\rightarrow} S^{3} \stackrel{i}{\rightarrow} S U(3) ;$

(e) the map $S U(3) \stackrel{\pi}{\rightarrow} S^{5}$ induces an injection on $\pi_{8}$;

(f) any map $f: S^{10} \rightarrow S U(3)$ has the property that $S^{11} \stackrel{\eta}{\rightarrow} S^{10} \stackrel{f}{\rightarrow} S U(3)$ is null homotopic.

The following lemma is surely well known, but an explicit proof seems to be difficult to find. It can be deduced from [HK1, Theorem 1]; we give a direct proof.

Lemma 5.2. There is an isomorphism $\left[\Sigma \mathbb{C} P^{2}, \Omega S U(3)\right] \cong \mathbb{Z} / 3 \mathbb{Z}$.

Proof. It is equivalent to adjoint and calculate $\left[\Sigma^{2} \mathbb{C} P^{2}, S U(3)\right]$. Consider the homotopy cofibration sequence

$$
S^{3} \stackrel{a}{\rightarrow} \Sigma \mathbb{C} P^{2} \stackrel{b}{\rightarrow} S^{5} \stackrel{\eta}{\rightarrow} S^{4}
$$

where $a$ is the inclusion of the bottom cell and $b$ is the pinch map to the top cell. This induces an exact sequence

$$
\pi_{5}(S U(3)) \stackrel{\eta^{*}}{\longrightarrow} \pi_{6}(S U(3)) \stackrel{b^{*}}{\longrightarrow}\left[\Sigma^{2} \mathbb{C} P^{2}, S U(3)\right] \stackrel{a^{*}}{\longrightarrow} \pi_{4}(S U(3)) .
$$

In the calculations that follow we use Lemma 5.1 freely. Since $\pi_{4}(S U(3))=0, b_{*}$ is an epimorphism. We have $\pi_{6}(S U(3)) \cong \mathbb{Z} / 6 \mathbb{Z} \cong \mathbb{Z} / 2 \mathbb{Z} \oplus \mathbb{Z} / 3 \mathbb{Z}$. The $\mathbb{Z} / 2 \mathbb{Z}$ summand of $\pi_{6}(S U(3))$ is represented by a map which factors through $\eta$, implying it is in the image of $\eta^{*}$. Thus $b^{*}$ is trivial on the $\mathbb{Z} / 2 \mathbb{Z}$ summand. On the other hand, since $\eta$ represents a homotopy class of order $2, \eta^{*}$ is zero on the 3 -primary component. Thus $b^{*}$ is an injection on the 3 -primary component. Hence $\left[\Sigma \mathbb{C} P^{2}, \Omega S U(3)\right]$ $\cong \mathbb{Z} / 3 \mathbb{Z}$.

Next, consider the homotopy cofibration sequence

$$
S^{3} \stackrel{f}{\rightarrow} \bigvee_{i=1}^{d} S^{2} \stackrel{i}{\rightarrow} M \stackrel{q}{\rightarrow} S^{4} \stackrel{\Sigma f}{\rightarrow} \bigvee_{i=1}^{d} S^{3} .
$$


Applying the functor $\operatorname{Map}_{k}^{*}(, B S U(3))$, we obtain a homotopy fibration sequence

$$
\begin{aligned}
& \prod_{i=1}^{d} \Omega^{2} S U(3) \stackrel{(\Sigma f)^{*}}{\longrightarrow} \Omega_{0}^{3} S U(3) \stackrel{q^{*}}{\longrightarrow} \operatorname{Map}_{k}^{*}(M, B S U(3)) \\
& \stackrel{i^{*}}{\rightarrow} \prod_{i=1}^{d} \Omega S U(3) \stackrel{f^{*}}{\longrightarrow} \Omega^{2} S U(3) .
\end{aligned}
$$

Lemma 5.3. With respect to (3), the following hold:

(a) 3-locally, $q^{*}$ induces an injection on $\pi_{3}$;

(b) integrally, $q^{*}$ induces an injection on $\pi_{5}$;

(c) integrally, $i^{*}$ induces an injection on $\pi_{4}$.

Proof. For part (a), apply $\pi_{3}$ to (3). As mentioned in Section 3, 3-locally $(\Sigma f)^{*}$ is null homotopic. Thus $q^{*}$ induces a 3 -local injection. For part (b), apply $\pi_{5}$ to (3). By Lemma $5.1, \pi_{5}\left(\prod_{i=1}^{d} \Omega^{2} S U(3)\right) \cong 0$. Thus by exactness, $\pi_{5}\left(q^{*}\right)$ is an injection. Part (c) is a similar exactness argument, using the fact from Lemma 5.1 that $\pi_{4}\left(\Omega_{0}^{3} S U(3)\right)=0$.

Lemma 5.3 holds for all simply-connected, closed 4-manifolds $M$. In Lemma 5.4 we begin to distinguish between Spin and non-Spin manifolds.

Lemma 5.4. Localize at 2 . With respect to (3), the following hold:

(a) if $M$ is a Spin manifold then $\pi_{3}\left(\operatorname{Map}_{k}^{*}(M, B S U(3))\right) \cong \mathbb{Z} / 2 \mathbb{Z}$ and $q^{*}$ induces an isomorphism on $\pi_{3}$;

(b) if $M$ is a non-Spin manifold then $\pi_{3}\left(\operatorname{Map}_{k}^{*}(M, B S U(3))\right) \cong 0$.

Proof. Apply $\pi_{3}$ to (3). This is equivalent to applying the functor $\left[S^{3},\right]$. Doing so and adjointing, we obtain an exact sequence

$$
\begin{aligned}
\cdots \rightarrow\left[\bigvee_{i=1}^{d} S^{6}, B S U(3)\right] \stackrel{\widetilde{f}}{\rightarrow}\left[S^{7}, B S U(3)\right] & \stackrel{\widetilde{q}}{\rightarrow}\left[\Sigma^{3} M, B S U(3)\right] \\
& \stackrel{\widetilde{i}}{\rightarrow}\left[\bigvee_{i=1}^{d} S^{5}, B S U(3)\right] \rightarrow \cdots
\end{aligned}
$$

By Lemma $5.1, \pi_{5}(B S U(3))=0$ and $\pi_{7}(B S U(3)) \cong \mathbb{Z} / 2 \mathbb{Z}$. Thus we either have $\left[\Sigma^{3} M, B S U(3)\right] \cong \mathbb{Z} / 2 \mathbb{Z}$ and $\widetilde{q}$ is an isomorphism, or $\left[\Sigma^{3} M, B S U(3)\right] \cong 0$. Observe as well that the map $\tilde{f}$ is induced by $S^{7} \stackrel{\Sigma^{4} f}{\longrightarrow} \bigvee_{i=1}^{d} S^{6}$.

If $M$ is a Spin manifold then $\Sigma^{4} f$ is null homotopic, so $\tilde{f}$ is the zero map in (4), implying that $\widetilde{q}$ is an isomorphism and $\left[\Sigma^{3} M, B S U(3)\right] \cong \mathbb{Z} / 2 \mathbb{Z}$, which proves part (a). 
If $M$ is a non-Spin manifold then $\Sigma^{4} f$ is nontrivial. By the Hilton-Milnor Theorem, we have $\pi_{7}\left(\bigvee_{i=1}^{d} S^{6}\right) \cong \bigoplus_{i=1}^{d} \pi_{7}\left(S^{6}\right) \cong \bigoplus_{i=1}^{d} \mathbb{Z} / 2 \mathbb{Z}$, where each generator of $\pi_{7}\left(S^{6}\right)$ is represented by $\eta$. Since $\Sigma^{4} f$ represents a nontrivial class in $\pi_{7}\left(\bigvee_{i=1}^{d} S^{6}\right)$, we have $\Sigma^{4} f$ homotopic to a composite $S^{7} \stackrel{\Delta}{\longrightarrow} \bigvee_{\bar{i}=1}^{\bar{d}} S^{7} \stackrel{\bigvee_{\bar{i}=1}^{\bar{d}} \eta}{\longrightarrow} \bigvee_{\bar{i}=1}^{\bar{d}} S^{6} \hookrightarrow \bigvee_{i=1}^{d} S^{6}$ for some integer $1 \leq \bar{d} \leq d$, where $\Delta$ is the iterated diagonal map. Now define a map $\theta: \bigvee_{i=1}^{d} S^{6} \rightarrow B S U(3)$ by the composite $\bigvee_{i=1}^{d} S^{6} \rightarrow S^{6} \stackrel{\bar{c}}{\rightarrow} B S U(3)$, where the left map pinches to the wedge summand corresponding to $\bar{i}=1$, and $\bar{c}$ is the adjoint of the map $c$ representing the generator of $\pi_{5}(S U(3)) \cong \mathbb{Z}$. Observe that $\theta \circ \Sigma^{4} f$ is homotopic to the composite $S^{7} \stackrel{\eta}{\rightarrow} S^{6} \stackrel{\bar{c}}{\rightarrow} B S U(3)$, which by Lemma 5.1 represents the generator of $\pi_{7}(B S U(3)) \cong \mathbb{Z} / 2 \mathbb{Z}$. Therefore, in the context of (4), $\theta$ represents a nontrivial class in $\left[\mathrm{V}_{i=1}^{d} S^{6}, B S U(3)\right]$ and we have $\tilde{f}(\theta)=\theta \circ \Sigma^{4} f$ representing the generator of $\left[S^{7}, B S U(3)\right] \cong \mathbb{Z} / 2 \mathbb{Z}$. Thus $\tilde{f}$ is onto, implying that $\left[\Sigma^{3} M, B S U(3)\right] \cong 0$, which proves part (b).

In Lemma 5.3 we showed that the map $i^{*}$ in (3) induces an injection on $\pi_{4}$. As a consequence of Lemma 5.4, we are able to say more.

Corollary 5.5. Localize at 2. With respect to (3), the following hold:

(a) if $M$ is a Spin manifold then $i^{*}$ induces an isomorphism on $\pi_{4}$;

(b) if $M$ is a non-Spin manifold then $f^{*}$ induces an epimorphism on $\pi_{4}$.

Proof. Apply $\pi_{4}$ to (3). The corollary follows immediately from Lemma 5.4 and exactness.

\section{§6. An upper bound on the localized homotopy types of $\mathcal{G}_{k}(M)$ when $M$ is non-Spin}

We aim towards Proposition 6.9, which states that if $(12, k)=\left(12, k^{\prime}\right)$ then $\mathcal{G}_{k}(M) \simeq \mathcal{G}_{k^{\prime}}(M)$ after localizing rationally or at any prime. This is based on showing that the composite $S U(3) \stackrel{\partial_{1}}{\longrightarrow} \Omega_{0}^{3} S U(3) \stackrel{12}{\longrightarrow} \Omega_{0}^{3} S U(3) \stackrel{q^{*}}{\rightarrow} \operatorname{Map}_{k}^{*}(M, B S U(3))$ is null homotopic. The tricky part comes from the fact that $12 \circ \partial_{1}$ is not null homotopic. That is, the composition with $q^{*}$ plays a nontrivial role.

We first list some properties of $S U(3) \stackrel{\partial_{1}}{\longrightarrow} \Omega_{0}^{3} S U(3)$ determined in [HK2]. Let $\imath: \Sigma \mathbb{C} P^{2} \rightarrow S U(3)$ be the canonical inclusion.

Lemma 6.1. The following hold:

(a) the map $S U(3) \stackrel{\partial_{1}}{\longrightarrow} \Omega_{0}^{3} S U(3)$ has order 24 ;

(b) the composite $\Sigma \mathbb{C} P^{2} \stackrel{\imath}{\rightarrow} S U(3) \stackrel{\partial_{1}}{\rightarrow} \Omega_{0}^{3} S U(3)$ has order 24 ;

(c) the composite $S^{3} \hookrightarrow S U(3) \stackrel{\partial_{1}}{\longrightarrow} \Omega_{0}^{3} S U(3)$ has order 6 . 
Next, we state a result due to Mimura $[\mathrm{M}]$.

Lemma 6.2. The inclusion $\Sigma \mathbb{C} P^{2} \stackrel{\imath}{\rightarrow} S U(3)$ has a left homotopy inverse after three suspensions. Consequently, there is a homotopy decomposition $\Sigma^{3} S U(3) \simeq$ $\Sigma^{4} \mathbb{C} P^{2} \vee \Sigma^{3} S^{8}$.

Define the space $C$ and the map $b$ by the cofibration $S^{3} \rightarrow S U(3) \stackrel{b}{\rightarrow} C$. Observe that $C$ is a two-cell complex with cells in dimensions 5 and 8 . The decomposition in Lemma 6.2 implies that $\Sigma^{3} C \simeq \Sigma^{3} S^{5} \vee \Sigma^{3} S^{8}$. But the attaching map for the top cell of $C$ is in the stable range, so it is null homotopic. Thus $C \simeq S^{5} \vee S^{8}$.

We now begin a series of lemmas to identify the effect of composing $\partial_{1}$ with the $2^{\text {nd }}$ and $4^{\text {th }}$-power maps on $\Omega_{0}^{3} S U(3)$. We will work 2-locally. By Lemma $6.1(\mathrm{c})$, the composite $S^{3} \rightarrow S U(3) \stackrel{\partial_{1}}{\rightarrow} \Omega_{0}^{3} S U(3)$ has order 2 when localized at 2 . Thus we immediately obtain the following.

Lemma 6.3. Localize at 2. There is a homotopy commutative diagram

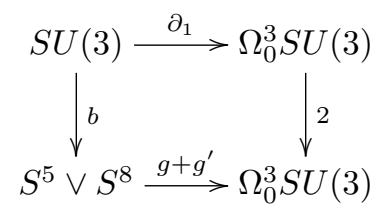

for some maps $g$ and $g^{\prime}$.

We now record some information about the maps $g$ and $g^{\prime}$.

Lemma 6.4. Let $g$ and $g^{\prime}$ be the maps in Lemma 6.3 and let $M$ be any simplyconnected 4-manifold. Then localized at 2 the following hold:

(a) the map $g$ represents a generator of $\pi_{5}\left(\Omega_{0}^{3} S U(3)\right) \cong \mathbb{Z} / 4 \mathbb{Z}$;

(b) the composite $S^{5} \stackrel{g}{\rightarrow} \Omega_{0}^{3} S U(3) \stackrel{q^{*}}{\rightarrow} \operatorname{Map}_{k}^{*}(M, B S U(3))$ has order 4 ;

(c) the map $g^{\prime}$ has order at most 2.

Proof. We first consider part (c). Since the $2^{\text {nd }}$-power map on $\Omega_{0}^{3} S U(3)$ is a 3 -fold loop map, we can adjoint the diagram in Lemma 6.3 to obtain a homotopy commutative diagram

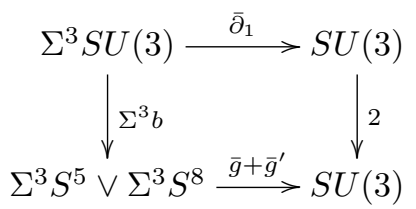


where $\bar{\partial}_{1}, \bar{g}$ and $\bar{g}^{\prime}$ are the adjoints of $\partial_{1}, g$ and $g^{\prime}$ respectively. By Lemma 6.2 , there is a map $t: \Sigma^{3} S^{8} \rightarrow \Sigma^{3} S U(3)$ such that the composite $\Sigma^{3} S^{8} \stackrel{t}{\rightarrow} \Sigma^{3} S U(3) \stackrel{\Sigma^{3} b}{\longrightarrow}$ $\Sigma^{3} S^{5} \vee \Sigma^{3} S^{8}$ is homotopic to the inclusion of the right wedge summand. By Lemma $5.1, \pi_{11}(S U(3)) \cong \mathbb{Z} / 4 \mathbb{Z}$. Thus $\bar{\partial}_{1} \circ t$ has order at most 4 . So $2 \circ \bar{\partial}_{1} \circ t$ has order at most 2 . The homotopy commutativity of the preceding diagram therefore implies that $\left(\bar{g}+\bar{g}^{\prime}\right) \circ \Sigma^{3} b \circ t$ has order at most 2. By definition of $t$, we have $\left(\bar{g}+\bar{g}^{\prime}\right) \circ b \circ t \simeq \bar{g}^{\prime}$. Thus $\bar{g}^{\prime}$ has order at most 2. Adjointing back, $g^{\prime}$ has order at most 2, proving part (c).

For part (a), by Lemma $6.1(\mathrm{a}), 2 \circ \partial_{1}$ has order 4 . So the homotopy commutativity of the diagram in Lemma 6.3 implies that $\left(g+g^{\prime}\right) \circ b$ has order 4 . We have just seen that $g^{\prime}$ has order at most 2, so $g$ must have order at least 4 . On the other hand, by Lemma $5.1, \pi_{5}\left(\Omega_{0}^{3} S U(3)\right) \cong \mathbb{Z} / 4 \mathbb{Z}$, so the order of $g$ is exactly 4 and $g$ represents a generator.

For part (b), Lemma 5.3 states that $q^{*}$ induces an injection on $\pi_{5}$. So as $g$ has order $4, q^{*} \circ g$ does as well.

Note that when $S U(3) \stackrel{b}{\rightarrow} S^{5} \vee S^{8}$ is pinched to $S^{5}$ we obtain the canonical map $S U(3) \stackrel{\pi}{\rightarrow} S^{5}$. By Lemma 6.4, the map $g^{\prime}$ in Lemma 6.3 has order 2. So we can immediately refine the diagram in Lemma 6.3 to the following.

Lemma 6.5. Localized at 2 , there is a homotopy commutative diagram

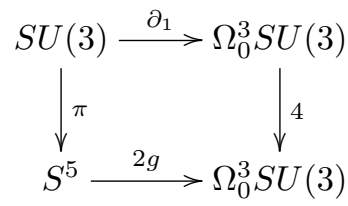

where $g$ represents a generator of $\pi_{5}\left(\Omega_{0}^{3} S U(3)\right) \cong \mathbb{Z} / 4 \mathbb{Z}$.

In what follows, we require a slightly different formulation of the diagram in Lemma 6.5.

Lemma 6.6. Let $t$ be an integer such that $(2, t)=1$. Then localized at 2 there is a homotopy commutative diagram

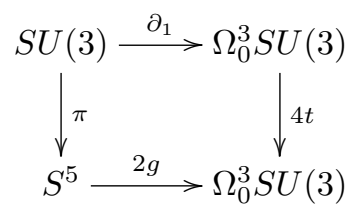

where $g$ represents a generator of $\pi_{5}\left(\Omega_{0}^{3} S U(3)\right) \cong \mathbb{Z} / 4 \mathbb{Z}$. 
Proof. Lemma 6.3 still holds when multiplication by 2 has been replaced with multiplication by $2 t$, although the choices of $g$ and $g^{\prime}$ may be different. Nevertheless, their orders remain the same as before since the argument in Lemma 6.4 goes through without change. The diagram asserted by the lemma then follows as $g^{\prime}$ has order 2 .

Next, we want to relate the map $g$ in Lemma 6.5 with $q^{*}$. For this, we specialize to the case of a non-Spin manifold.

Lemma 6.7. Let $M$ be a simply-connected non-Spin 4-manifold. Then localized at 2 there is a homotopy commutative diagram

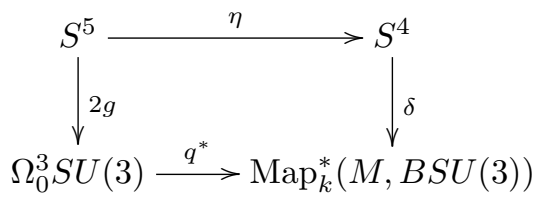

where $g$ is the map in Lemma 6.5 and $\delta$ has the property that the composite $S^{4} \stackrel{\delta}{\rightarrow}$ $\operatorname{Map}_{k}^{*}(M, B S U(3)) \stackrel{i^{*}}{\rightarrow} \prod_{i=1}^{d} \Omega S U(3)$ is divisible by 2 .

Proof. We begin by defining some maps. Consider the homotopy fibration sequence

$$
\Omega_{0}^{3} S U(3) \stackrel{q^{*}}{\rightarrow} \operatorname{Map}_{k}^{*}(M, B S U(3)) \stackrel{i^{*}}{\rightarrow} \prod_{i=1}^{d} \Omega S U(3) \stackrel{f^{*}}{\rightarrow} \Omega^{2} S U(3) .
$$

Since $M$ is non-Spin, by Corollary 5.5(b), there is a map $\gamma: S^{4} \rightarrow \prod_{i=1}^{d} \Omega S U(3)$ such that $f^{*} \circ \gamma$ represents the generator of $\pi_{4}\left(\Omega^{2} S U(3)\right) \cong \mathbb{Z} / 2 \mathbb{Z}$. Since this group has order $2, f^{*} \circ \gamma$ extends to a map $e: P^{5}(2) \rightarrow \Omega^{2} S U(3)$, where $P^{m}(2)$ is the mod-2 Moore space of dimension $m$. From the extension of $f^{*} \circ \gamma$ to $e$ we obtain a homotopy commutative diagram

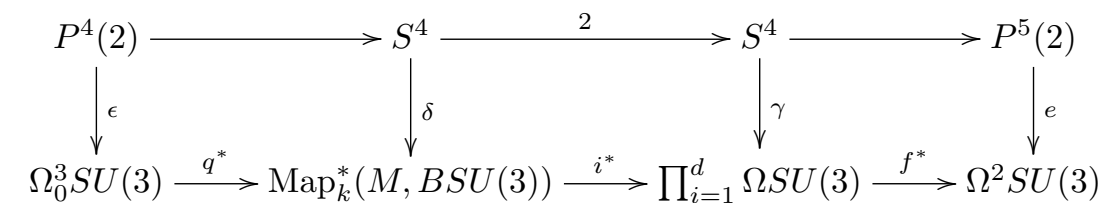

where the top row is a homotopy cofibration, the bottom row is a homotopy fibration, and the right square induces the maps $\delta$ and $\epsilon$. Note that there may be different choices of $\delta$ and $\epsilon$, but the Peterson-Stein formulas imply that these maps can be chosen so that $\epsilon$ is the adjoint of $e$. Doing so implies that $\epsilon$ is an extension of a map $S^{3} \rightarrow \Omega_{0}^{3} S U(3)$ representing the generator of $\pi_{3}\left(\Omega_{0}^{3} S U(3)\right) \cong \mathbb{Z} / 2 \mathbb{Z}$. 
Next, since $S^{4} \stackrel{\eta}{\rightarrow} S^{3}$ has order 2 , there is a homotopy commutative diagram

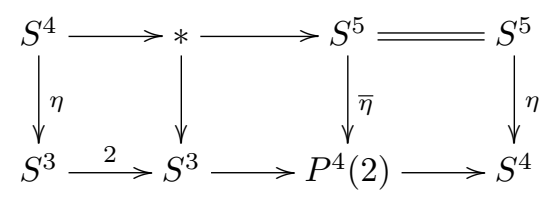

which defines the map $\bar{\eta}$. There may be different choices of $\bar{\eta}$ but any choice will do.

We claim that the composite $S^{5} \stackrel{\bar{\eta}}{\rightarrow} P^{4}(2) \stackrel{\epsilon}{\rightarrow} \Omega_{0}^{3} S U(3)$ is homotopic to $\pm 2 g$, where $g$ represents the generator of $\pi_{5}\left(\Omega_{0}^{3} S U(3)\right) \cong \mathbb{Z} / 4 \mathbb{Z}$. If so then precomposing the left square in (5) with $\bar{\eta}$ gives a homotopy commutative diagram

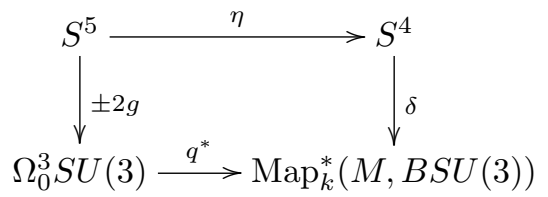

Replacing $\delta$ by $-\delta$ if necessary to adjust $\pm 2 g$ to $2 g$, we obtain the homotopy commutative diagram asserted by the lemma.

It remains to show that $\epsilon \circ \bar{\eta} \simeq \pm 2 g$. Since $g$ has order 4 , it is equivalent to show that $\epsilon \circ \bar{\eta}$ has order 2. Adjointing, it is equivalent to show that the composite $S^{8} \stackrel{\Sigma^{3} \bar{\eta}}{\longrightarrow} P^{7}(2) \stackrel{\epsilon^{\prime}}{\rightarrow} S U(3)$ has order 2 , where $\epsilon^{\prime}$ is the adjoint of $\epsilon$. Since $\epsilon$ is an extension of a map representing the generator of $\pi_{3}\left(\Omega_{0}^{3} S U(3)\right) \cong \mathbb{Z} / 2 \mathbb{Z}$, its adjoint $\epsilon^{\prime}$ is an extension of a map representing the generator of $\pi_{6}(S U(3))$. On the other hand, by Lemma 5.1, this generator is represented by the composite $S^{6} \stackrel{\nu^{\prime}}{\rightarrow} S^{3} \stackrel{i}{\rightarrow} S U(3)$. Thus there is a homotopy commutative diagram

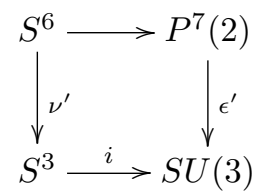

This induces a homotopy commutative diagram

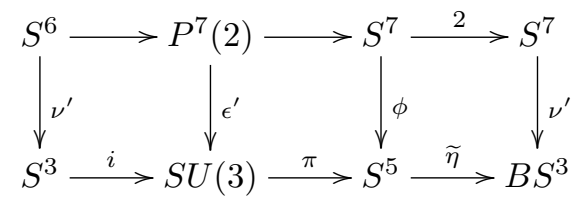

where the top row is a homotopy cofibration sequence, the bottom row is a homotopy fibration sequence, $\widetilde{\eta}$ represents the generator of $\pi_{5}\left(B S^{3}\right) \cong \pi_{4}\left(S^{3}\right) \cong \mathbb{Z} / 2 \mathbb{Z}$, 
and $\phi$ is some induced map. Adjointing the right square in (6) we obtain $\eta \circ \phi \simeq \nu^{\prime} \circ 2$ in $\pi_{6}\left(S^{3}\right) \cong \mathbb{Z} / 4 \mathbb{Z}$. Since $\nu^{\prime}$ represents a generator of this group, $\nu^{\prime} \circ 2$ is nontrivial. Thus $\phi$ is nontrivial, so as $\pi_{5}\left(S^{3}\right) \cong \mathbb{Z} / 2 \mathbb{Z}$ is generated by $\eta^{2}$, we have $\phi \simeq \eta^{2}$. Now consider the middle square of (6). By definition of $\bar{\eta}$, the map $S^{8} \stackrel{\Sigma^{3} \bar{\eta}}{\longrightarrow} P^{7}(2)$ has the property that when composed with the pinch map $P^{7}(2) \rightarrow S^{7}$ we obtain $\eta$. Thus the middle square of (6) implies that $\pi \circ \epsilon^{\prime} \circ \Sigma^{3} \bar{\eta} \simeq \eta^{3}$. In particular, as $\eta^{3}$ is nontrivial of order 2, we see that $\pi \circ \epsilon^{\prime} \circ \Sigma^{3} \bar{\eta}$ is nontrivial of order 2. Finally, by Lemma 5.1 , the map $\pi$ induces an injection on $\pi_{8}$, so $\epsilon^{\prime} \circ \Sigma^{3} \bar{\eta}$ is nontrivial of order 2 , as required.

Next, we combine the previous lemmas to prove a key null homotopy.

Proposition 6.8. Let $t$ be an integer such that $(2, t)=1$ and let $M$ be a simplyconnected non-Spin 4-manifold. Then localized at 2 the composite $S U(3) \stackrel{\partial_{1}}{\longrightarrow}$ $\Omega_{0}^{3} S U(3) \stackrel{4 t}{\longrightarrow} \Omega_{0}^{3} S U(3) \stackrel{q^{*}}{\rightarrow} \operatorname{Map}_{k}^{*}(M, B S U(3))$ is null homotopic.

Proof. First observe that there is a homotopy pushout diagram

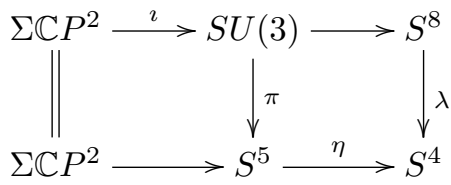

which defines the map $\lambda$. By [To2], $\pi_{8}\left(S^{4}\right)=\mathbb{Z} / 2 \mathbb{Z} \oplus \mathbb{Z} / 2 \mathbb{Z}$, where the generators are $\nu \circ \eta$ and $\Sigma \nu^{\prime} \circ \eta$. Thus $\lambda$ is homotopic to a composite $S^{8} \stackrel{\eta}{\rightarrow} S^{7} \stackrel{v}{\rightarrow} S^{4}$ where $v$ is some sum of $\nu$ and $\Sigma \nu^{\prime}$.

Now consider the diagram

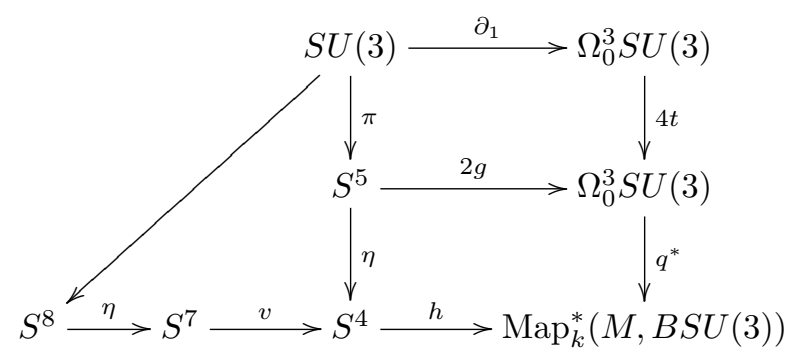

The left triangle homotopy commutes by the previous paragraph. The upper right square homotopy commutes by Lemma 6.6 and the lower right square homotopy commutes by Lemma 6.7. We will show that the composite along the bottom row is null homotopic. If so, then the lower direction around the diagram is null homotopic, implying that the upper direction around the diagram is also null homotopic. 
But the upper direction around the diagram is exactly as in the statement of the lemma.

It remains to show that $h \circ v \circ \eta$ is null homotopic. First consider $S^{7} \stackrel{v}{\rightarrow} S^{4} \stackrel{h}{\rightarrow}$ $\operatorname{Map}_{k}^{*}(M, B S U(3))$. Apply $\pi_{7}$ to the homotopy fibration sequence

$$
\prod_{i=1}^{d} \Omega^{2} S U(3) \rightarrow \Omega_{0}^{3} S U(3) \stackrel{q^{*}}{\rightarrow} \operatorname{Map}_{k}^{*}(M, B S U(3)) \stackrel{i^{*}}{\rightarrow} \prod_{i=1}^{d} \Omega S U(3) \rightarrow \Omega^{2} S U(3) .
$$

By Lemma 5.1, we obtain a short exact sequence

$$
0 \rightarrow \mathbb{Z} / 2 \mathbb{Z} \stackrel{\pi_{7}\left(q^{*}\right)}{\longrightarrow} \pi_{7}\left(\operatorname{Map}_{k}^{*}(M, B S U(3))\right) \stackrel{\pi_{7}\left(i^{*}\right)}{\longrightarrow} \bigoplus_{i=1}^{d} \mathbb{Z} / 4 \mathbb{Z} \rightarrow 0 .
$$

In general, suppose that $0 \rightarrow G_{1} \stackrel{f}{\rightarrow} G_{2} \stackrel{g}{\rightarrow} G_{3} \rightarrow 0$ is a short exact sequence of groups. Let $x \in G_{2}$ and suppose that $g(x)=2 y$. Since $g$ is onto, there is an element $x^{\prime} \in G_{2}$ such that $g\left(x^{\prime}\right)=y$. Then $g\left(x-2 x^{\prime}\right)=0$ so $x-2 x^{\prime}$ lifts to $z \in G_{1}$. Thus $x=2 x^{\prime}+f(z)$. In our case, consider $x=h \circ v$ and the short exact sequence (7). By Lemma 6.7, $i^{*} \circ h \simeq 2 \gamma$ for some map $\gamma$, so $i^{*} \circ h \circ v \simeq 2 \gamma \circ v$. That is, $g(x)=2 y$ where $g=\pi_{7}\left(i^{*}\right)$ and $y=\gamma \circ v$. So the general argument above shows that $x=h \circ v \simeq 2 x^{\prime}+q^{*} \circ z$, where $z$ represents a class in $\pi_{7}\left(\Omega_{0}^{3} S U(3)\right)$.

Now consider $h \circ v \circ \eta$. Since $\eta$ has order 2, we have $h \circ v \circ \eta \simeq 2 x^{\prime} \circ \eta+$ $q^{*} \circ z \circ \eta \simeq q^{*} \circ z \circ \eta$. The map $q^{*} \circ z$ represents a class in $\pi_{7}\left(\Omega_{0}^{3} S U(3)\right) \cong \mathbb{Z} / 2 \mathbb{Z}$. By Lemma 5.1(d), $q^{*} \circ z \circ \eta$ is null homotopic. Hence $h \circ v \circ \eta \simeq q^{*} \circ z \circ \eta$ is null homotopic, as claimed.

Finally, we are able to give some information on the homotopy types of $S U(3)$ gauge groups over a simply-connected non-Spin 4-manifold.

Proposition 6.9. Let $M$ be a simply-connected non-Spin 4-manifold. If $(12, k)=$ $\left(12, k^{\prime}\right)$ then there is a homotopy equivalence $\mathcal{G}_{k}(M) \simeq \mathcal{G}_{k^{\prime}}(M)$ after localizing rationally or at any prime.

Proof. By Lemma 6.1(a), the map $S U(3) \stackrel{\partial_{1}}{\longrightarrow} \Omega_{0}^{3} S U(3)$ has order 24. So Lemma 4.2 implies that if $(24, k)=\left(24, k^{\prime}\right)$ then there is a homotopy equivalence $\mathcal{G}_{k}(M) \simeq$ $\mathcal{G}_{k^{\prime}}(M)$ after localizing rationally or at any prime. However, we need to do a bit better than this.

Since 24 has eight integer divisors, there are at most eight possibilities for the homotopy types of $\mathcal{G}_{k}(M)$ after localizing rationally or at an odd prime. Equivalently, there are at most four 2-local homotopy types of $\mathcal{G}_{k}(M)$, depending on the value of $(8, k)$, and at most two 3 -local homotopy types, depending on the value of $(3, k)$. We will show that in the 2 -local case, the types corresponding to $(8, k)=4$ 
and $(8, k)=8$ are identified. If so, then $(12, k)=\left(12, k^{\prime}\right)$ implies that $\mathcal{G}_{k}(M) \simeq$ $\mathcal{G}_{k^{\prime}}(M)$ after localizing rationally or at any prime, proving the proposition.

Localize at 2 and consider the homotopy fibration $\mathcal{G}_{k}(M) \rightarrow S U(3) \stackrel{\partial_{k}^{M}}{\longrightarrow}$ $\operatorname{Map}_{k}^{*}(M, B S U(3))$. By definition, $\partial_{k}^{M}$ is the composite $S U(3) \stackrel{\partial_{k}}{\longrightarrow} \Omega_{0}^{3} S U(3) \stackrel{q^{*}}{\longrightarrow}$ $\operatorname{Map}_{k}^{*}(M, B S U(3))$, and by Lemma $2.1, \partial_{k} \simeq k \circ \partial_{1}$. If $(8, k)=8$ then $k=8 t$. By Lemma 6.1(a), $\partial_{1}$ has order 8 , so $\partial_{k}=k \circ \partial_{1} \simeq 8 t \circ \partial_{1}$ is null homotopic. Thus $\partial_{k}^{M}$ is null homotopic, implying that $\mathcal{G}_{k}(M) \simeq S U(3) \times \operatorname{Map}_{k}^{*}(M, B S U(3))$. If $(8, k)=4$ then $k=4 t$ for some integer $t$ satisfying $(2, t)=1$. Thus $\partial_{k}^{M} \simeq q^{*} \circ k \circ \partial_{1} \simeq$ $q^{*} \circ 4 t \circ \partial_{1}$. By Proposition 6.8, $q^{*} \circ 4 t \circ \partial_{1}$ is null homotopic. Hence $\partial_{k}^{M}$ is null homotopic, implying that $\mathcal{G}_{k}(M) \simeq S U(3) \times \operatorname{Map}_{k}^{*}(M, B S U(3))$. Summarizing, if $(8, k)=8$ or $(8, k)=4$ then the homotopy types of the corresponding gauge groups $\mathcal{G}_{k}(M)$ have been identified as $S U(3) \times \operatorname{Map}_{k}^{*}(M, B S U(3))$.

\section{$\S 7$. A lower bound on the number of homotopy types of $\mathcal{G}_{k}(M)$}

In this section we start with a homotopy equivalence $\mathcal{G}_{k}(M) \simeq \mathcal{G}_{k^{\prime}}(M)$ and show that $(24, k)=\left(24, k^{\prime}\right)$ if $M$ is Spin or $(12, k)=\left(12, k^{\prime}\right)$ if $M$ is non-Spin. The key tool is an analysis of the composite $\Sigma \mathbb{C} P^{2} \stackrel{\imath}{\rightarrow} S U(3) \stackrel{\partial_{1}}{\rightarrow} \Omega_{0}^{3} S U(3) \stackrel{q^{*}}{\rightarrow}$ $\operatorname{Map}_{k}^{*}(M, B S U(3))$.

Lemma 7.1. Let $M$ be a simply-connected 4-manifold. Consider the composite $\Sigma \mathbb{C} P^{2} \stackrel{\imath}{\rightarrow} S U(3) \stackrel{\partial_{1}}{\longrightarrow} \Omega_{0}^{3} S U(3) \stackrel{q^{*}}{\rightarrow} \operatorname{Map}_{k}^{*}(M, B S U(3))$. The following hold:

(a) if $M$ is Spin then $q^{*} \circ \partial_{1} \circ \imath$ has order 24 ;

(b) if $M$ is non-Spin then $q^{*} \circ \partial_{1} \circ \imath$ has order 12.

Proof. It suffices to show that $q^{*} \circ \partial_{1} \circ \imath$ has order 3 when localized at 3 , order 8 when localized at 2 in the Spin case, and order 4 when localized at 2 in the non-Spin case.

Localize at 3. By Lemma 6.1, $\partial_{1} \circ \imath$ has order 3 , so $q^{*} \circ \partial_{1} \circ \imath$ has order at most 3. On the other hand, by Lemma 6.1(c), the composition $S^{3} \rightarrow \Sigma \mathbb{C} P^{2} \stackrel{\imath}{\rightarrow}$ $S U(3) \stackrel{\partial_{1}}{\longrightarrow} \Omega_{0}^{3} S U(3)$ has order 3 , and by Lemma $5.3, q^{*}$ induces an injection on $\pi_{3}$. Thus $q^{*} \circ \partial_{1} \circ \imath$ is nontrivial, implying that its order is at least 3. Hence $q^{*} \circ \partial_{1} \circ \imath$ has order 3 .

Localize at 2 . There are two cases.

Case 1: $M$ is Spin. By Lemma 6.1, $\partial_{1} \circ \imath$ has order 8, so $q^{*} \circ \partial_{1} \circ \imath$ has order at most 8 . We wish to show that $q^{*} \circ \partial_{1} \circ \imath$ also has order at least 8 . Let $\bar{\pi}$ be the composite $\bar{\pi}: \Sigma \mathbb{C} P^{2} \stackrel{\imath}{\rightarrow} S U(3) \stackrel{\pi}{\rightarrow} S^{5}$. Equivalently, $\bar{\pi}$ is the pinch map to the top 
cell. Consider the diagram

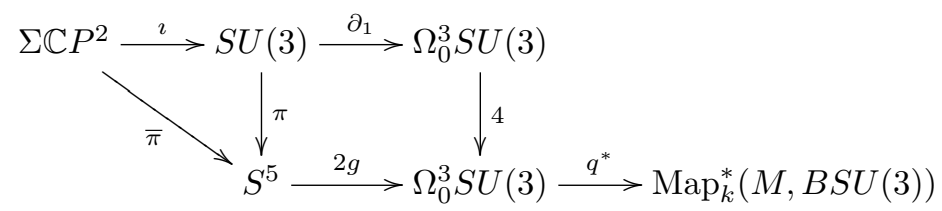

The left triangle homotopy commutes by definition of $\bar{\pi}$ and the middle square homotopy commutes by Lemma 6.5. To show that $q^{*} \circ \partial_{1} \circ \imath$ has order at least 8 it is equivalent to show that the upper direction around the diagram is nontrivial. We will do this by showing that the lower direction around the diagram is nontrivial.

Suppose otherwise, that $q^{*} \circ 2 g \circ \bar{\pi}$ is null homotopic. Consider the diagram

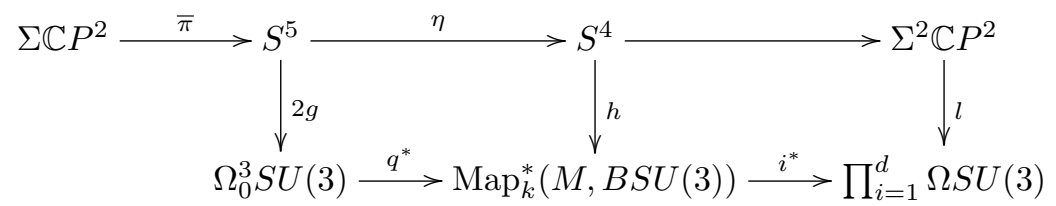

where $h$ and $l$ are to be defined momentarily. The null homotopy for $q^{*} \circ 2 g \circ \bar{\pi}$ implies that $q^{*} \circ 2 g$ extends along the homotopy cofibre of $\bar{\pi}$, making the left square homotopy commute for some choice of extension $h$. Since the top row is a homotopy cofibration and the bottom row is a homotopy fibration, the homotopy commutativity of the left square implies that $i^{*} \circ h$ extends along the homotopy cofibre of $\eta$, making the right square homotopy commute for some choice of extension $l$. Thus (8) homotopy commutes so we can begin to analyze its properties. Since $q^{*} \circ g$ has order 4 by Lemma 6.4(b), the composite $q^{*} \circ 2 g$ is nontrivial. Therefore the homotopy commutativity of the left square in (8) implies that: (i) $h$ is nontrivial and (ii) $h$ is not divisible by 2 (for if $h \simeq 2 h^{\prime}$ then $q^{*} \circ 2 g \simeq 2 h^{\prime} \circ \eta \simeq *$ because $\eta$ has order 2). By Lemma 5.5, $i^{*}$ induces an isomorphism on $\pi_{4}$. Therefore, $i^{*} \circ h$ is nontrivial and is not divisible by 2 . By Lemma 5.1 , there is a 2-local isomorphism $\pi_{4}\left(\prod_{i=1}^{d} \Omega S U(3)\right) \cong \bigoplus_{i=1}^{d} \mathbb{Z}_{(2)}$, and a generating set is represented by the composites $c_{i}: S^{4} \stackrel{\tilde{c}}{\rightarrow} \Omega S U(3) \hookrightarrow \prod_{i=1}^{d} \Omega S U(3)$ for $1 \leq i \leq d$, where $\tilde{c}$ is the adjoint of the map $c$ representing the generator of $\pi_{5}(S U(3)) \cong \mathbb{Z}_{(2)}$. Since $i^{*} \circ h$ is nontrivial and not divisible by 2 , we have $i^{*} \circ h \simeq \sum_{i=1}^{d} t_{i} c_{i}$ where each $t_{i} \in \mathbb{Z}_{(2)}$ and at least one $t_{i}$ - say $t_{i_{0}}$-is not divisible by 2 . By Lemma $5.1, \pi_{6}(\Omega S U(3)) \cong \bigoplus_{i=1}^{d} \mathbb{Z} / 2 \mathbb{Z}$, and a generating set is represented by the composites $c_{i} \circ \eta$. In particular, $t_{i_{0}} c_{i_{0}} \circ \eta$ is nontrivial, implying that $i^{*} \circ h \circ \eta$ is nontrivial. But this contradicts the homotopy commutativity of (8). Therefore the supposition that $q^{*} \circ 2 g \circ \bar{\pi}$ is null homotopic is false. Hence $q^{*} \circ g \circ \bar{\pi}$ has order at least 4 , as required. 
Case 2: $M$ is non-Spin. By Proposition 6.8, the composite $S U(3) \stackrel{\partial_{1}}{\rightarrow} \Omega_{0}^{3} S U(3) \stackrel{4}{\rightarrow}$ $\Omega_{0}^{3} S U(3) \stackrel{q^{*}}{\rightarrow} \operatorname{Map}_{k}^{*}(M, B S U(3))$ is null homotopic. Thus $q^{*} \circ \partial_{1} \circ \imath$ has order at most 4 . To show that $q^{*} \circ \partial_{1} \circ \imath$ has order at least 4 , we will show that $q^{*} \circ 2 \circ \partial_{1} \circ \imath$ is nontrivial. Consider the diagram

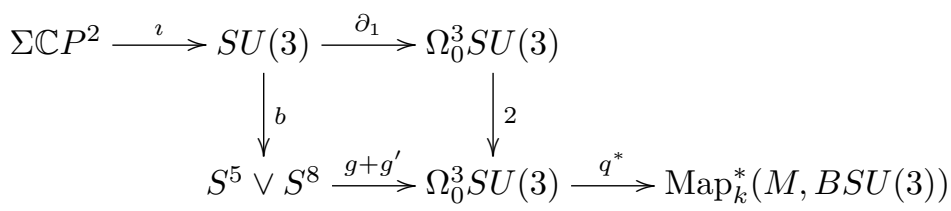

The square homotopy commutes by Lemma 6.3. We wish to show that the upper direction around the diagram is nontrivial. It is therefore equivalent to show that the lower direction around the diagram is nontrivial. By Lemma 5.1(a), there is a map $c: S^{5} \rightarrow S U(3)$ with the property that the composite $S^{5} \stackrel{c}{\rightarrow} S U(3) \stackrel{\pi}{\rightarrow} S^{5}$ has degree 2 . By [To1], $c$ can be chosen to factor as $S^{5} \stackrel{s}{\rightarrow} \Sigma \mathbb{C} P^{2} \stackrel{\imath}{\rightarrow} S U(3)$. Thus $q^{*} \circ\left(g+g^{\prime}\right) \circ b \circ \imath \circ s \simeq q^{*} \circ 2 g$. By Lemma 6.4(b), $q^{*} \circ g$ has order 4. Thus $q^{*} \circ 2 g$ is nontrivial, implying that $q^{*} \circ\left(g+g^{\prime}\right) \circ b \circ \imath$ is nontrivial. That is, the lower direction around the previous diagram is nontrivial, as required.

The map $\Omega_{0}^{3} S U(3) \stackrel{q^{*}}{\rightarrow} \operatorname{Map}_{k}^{*}(M, B S U(3))$ induces a map

$$
\bar{q}:\left[\Sigma \mathbb{C} P^{2}, \Omega_{0}^{3} S U(3)\right] \rightarrow\left[\Sigma \mathbb{C} P^{2}, \operatorname{Map}_{k}^{*}(M, B S U(3))\right] .
$$

The following lemma describes some of its 2-local properties.

Lemma 7.2. Let $M$ be a simply-connected 4-manifold. Localize at 2. The following hold:

(a) there is an isomorphism $\left[\Sigma \mathbb{C} P^{2}, \Omega_{0}^{3} S U(3)\right] \cong \mathbb{Z} / 8 \mathbb{Z}$ and a generator of order 8 is represented by the composite $\Sigma \mathbb{C} P^{2} \stackrel{\iota}{\rightarrow} S U(3) \stackrel{\partial_{1}}{\rightarrow} \Omega_{0}^{3} S U(3)$;

(b) if $M$ is Spin, $\left[\Sigma \mathbb{C} P^{2}, \Omega_{0}^{3} S U(3)\right] \stackrel{\bar{q}}{\rightarrow}\left[\Sigma \mathbb{C} P^{2}, \operatorname{Map}_{k}^{*}(M, B S U(3))\right]$ is an isomorphism;

(c) if $M$ is non-Spin, then $\left[\Sigma \mathbb{C} P^{2}, \operatorname{Map}_{k}^{*}(M, B S U(3))\right] \cong \mathbb{Z} / 4 \mathbb{Z}$, a representative of the generator is the composite $\Sigma \mathbb{C} P^{2} \stackrel{\imath}{\rightarrow} S U(3) \stackrel{\partial_{1}}{\rightarrow} \Omega_{0}^{3} S U(3) \stackrel{q^{*}}{\rightarrow}$ $\operatorname{Map}_{k}^{*}(M, B S U(3))$, and $\bar{q}$ induces the reduction map $\mathbb{Z} / 8 \mathbb{Z} \rightarrow \mathbb{Z} / 4 \mathbb{Z}$.

Proof. Part (a) is a refinement of Lemma 6.1(a), and was proved in [HK2].

For parts (b) and (c), first observe that if the functor $\left[\Sigma \mathbb{C} P^{2}, \quad\right]$ is applied to the homotopy fibration $\Omega_{0}^{3} S U(3) \stackrel{q^{*}}{\rightarrow} \operatorname{Map}_{k}^{*}(M, B S U(3)) \stackrel{i^{*}}{\rightarrow} \prod_{i=1}^{d} \Omega S U(3)$ we 
obtain an exact sequence

$$
\left[\Sigma \mathbb{C} P^{2}, \Omega_{0}^{3} S U(3)\right] \stackrel{\bar{q}}{\rightarrow}\left[\Sigma \mathbb{C} P^{2}, \operatorname{Map}_{k}^{*}(M, B S U(3))\right] \stackrel{i^{*}}{\rightarrow}\left[\Sigma \mathbb{C} P^{2}, \prod_{i=1}^{d} \Omega S U(3)\right] .
$$

By Lemma 5.2, $\left[\Sigma \mathbb{C} P^{2}, \Omega S U(3)\right] \cong 0$ (2-locally), so $\bar{q}$ is an epimorphism.

For part (b), as $M$ is Spin, Lemma 7.1(a) states that $q^{*} \circ \partial_{1} \circ \imath$ has order 8 , the same as $\partial_{1} \circ \imath$. Since $\partial_{1} \circ \imath$ represents a generator of $\left[\Sigma \mathbb{C} P^{2}, \Omega_{0}^{3} S U(3)\right]$, the map $\bar{q}$ is therefore an injection. Since $\bar{q}$ is also an epimorphism, it is an isomorphism.

For part (c), as $M$ is non-Spin, Lemma 7.1(b) states that $q^{*} \circ \partial_{1} \circ \imath$ has order 4. That is, $\bar{q}\left(\partial_{1} \circ \imath\right)$ has order 4 . Since $\partial_{1} \circ \imath$ represents a generator in $\left[\Sigma \mathbb{C} P^{2}, \Omega_{0}^{3} S U(3)\right] \cong \mathbb{Z} / 8 \mathbb{Z}$, the image of $\bar{q}$ is $\mathbb{Z} / 4 \mathbb{Z}$. As $\bar{q}$ is an epimorphism, we therefore have $\left[\Sigma \mathbb{C} P^{2}, \operatorname{Map}_{k}^{*}(M, B S U(3))\right] \cong \mathbb{Z} / 4 \mathbb{Z}$, a representative of the generator is $q^{*} \circ \partial_{1} \circ \imath$, and $\bar{q}$ is the reduction map.

We now extract some 2-primary information from a homotopy equivalence $\mathcal{G}_{k}(M) \simeq \mathcal{G}_{k^{\prime}}(M)$. Consider the homotopy fibration diagram

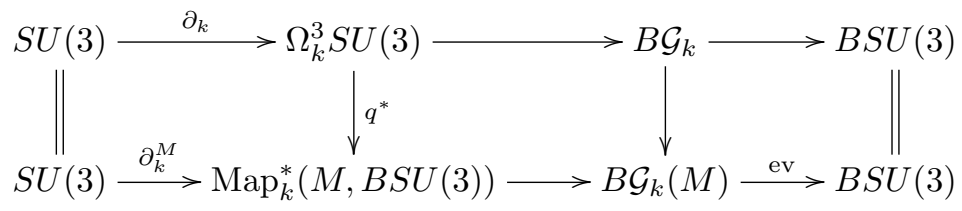

Apply the functor $\left[\Sigma \mathbb{C} P^{2}, \quad\right]$. Note that the canonical map $B S U(3) \rightarrow B S U(\infty)$ is 6-connected, implying that there are isomorphisms

$$
\left[\Sigma \mathbb{C} P^{2}, B S U(3)\right] \cong\left[\Sigma \mathbb{C} P^{2}, B S U(\infty)\right] \cong \widetilde{K}^{0}\left(\Sigma \mathbb{C} P^{2}\right)=0
$$

Thus we obtain a commutative diagram of exact sequences

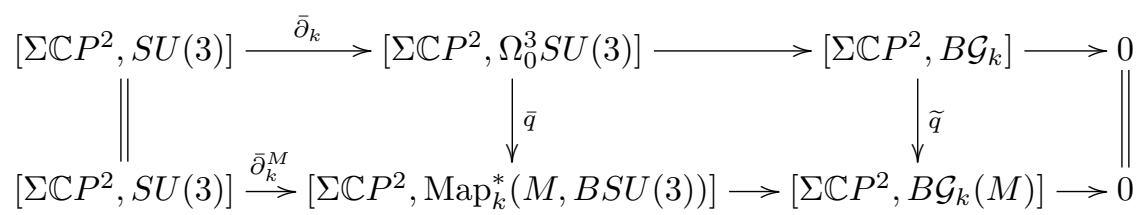

Lemma 7.3. Suppose there is a homotopy equivalence $\mathcal{G}_{k}(M) \simeq \mathcal{G}_{k^{\prime}}(M)$. The following hold:

(a) if $M$ is Spin then $(8, k)=\left(8, k^{\prime}\right)$;

(b) if $M$ is non-Spin then $(4, k)=\left(4, k^{\prime}\right)$.

Proof. The homotopy equivalence $\mathcal{G}_{k}(M) \simeq \mathcal{G}_{k^{\prime}}(M)$ implies that there is an isomorphism of sets $\left[\Sigma \mathbb{C} P^{2}, B \mathcal{G}_{k}(M)\right] \cong\left[\Sigma \mathbb{C} P^{2}, B \mathcal{G}_{k^{\prime}}(M)\right]$. Localize at 2 . 
If $M$ is Spin then Lemma 7.2(b) states that $\bar{q}$ is an isomorphism. A diagram chase in (9) therefore implies that $\widetilde{q}$ is an isomorphism. Thus there is an isomorphism of sets $\left[\Sigma \mathbb{C} P^{2}, B \mathcal{G}_{k}\right] \cong\left[\Sigma \mathbb{C} P^{2}, B \mathcal{G}_{k^{\prime}}\right]$. In [HK2] it is shown that if the latter isomorphism exists then $(8, k)=\left(8, k^{\prime}\right)$, proving part (a).

If $M$ is non-Spin then by Lemma $7.2(\mathrm{c}),\left[\Sigma \mathbb{C} P^{2}, \operatorname{Map}_{k}^{*}(M, B S U(3))\right] \cong \mathbb{Z} / 4 \mathbb{Z}$ and a generator is represented by the composite $\Sigma \mathbb{C} P^{2} \stackrel{\imath}{\rightarrow} S U(3) \stackrel{\partial_{1}}{\rightarrow} \Omega_{0}^{3} S U(3) \stackrel{q^{*}}{\rightarrow}$ $\operatorname{Map}_{k}^{*}(M, B S U(3))$. By Corollary 2.2, $\partial_{k} \simeq k \circ \partial_{1}$ and by its definition, $\partial_{k}^{M}=q^{*} \circ \partial_{k}$, so $\partial_{k}^{M} \simeq q^{*} \circ k \circ \partial_{1}$. Thus $\operatorname{Im} \bar{\partial}_{k}^{M} \cong \mathbb{Z} /(4 /(4, k)) \mathbb{Z}$, implying that coker $\bar{\partial}_{k}^{M} \cong$ $\mathbb{Z} /(4, k) \mathbb{Z}$. On the other hand, observe that the exactness of the bottom row in (9) implies that $\left[\Sigma \mathbb{C} P^{2}, B \mathcal{G}_{k}(M)\right]$ is the cokernel of $\bar{\partial}_{k}^{M}$. Thus $\left[\Sigma \mathbb{C} P^{2}, B \mathcal{G}_{k}(M)\right] \cong$ $\mathbb{Z} /(4, k) \mathbb{Z}$. Similarly, $\left[\Sigma \mathbb{C} P^{2}, B \mathcal{G}_{k^{\prime}}(M)\right] \cong \mathbb{Z} /\left(4, k^{\prime}\right) \mathbb{Z}$. By assumption, $\mathcal{G}_{k}(M) \simeq$ $\mathcal{G}_{k^{\prime}}(M)$, so there is a string of isomorphisms $\mathbb{Z} /(4, k) \mathbb{Z} \cong\left[\Sigma \mathbb{C} P^{2}, B \mathcal{G}_{k}(M)\right] \cong$ $\left[\Sigma \mathbb{C} P^{2}, B \mathcal{G}_{k^{\prime}}(M)\right] \cong \mathbb{Z} /\left(4, k^{\prime}\right) \mathbb{Z}$. This occurs if and only if $(4, k)=\left(4, k^{\prime}\right)$.

Next, we move to the 3-primary case.

Lemma 7.4. Suppose there is a homotopy equivalence $\mathcal{G}_{k}(M) \simeq \mathcal{G}_{k^{\prime}}(M)$. Then $(3, k)=\left(3, k^{\prime}\right)$.

Proof. The homotopy equivalence $\mathcal{G}_{k}(M) \simeq \mathcal{G}_{k^{\prime}}(M)$ implies that there is an isomorphism $\pi_{4}\left(\mathcal{G}_{k}(M)\right) \cong \pi_{4}\left(\mathcal{G}_{k^{\prime}}(M)\right)$. Localized at 3 , Theorem 3.1 states that there is a homotopy equivalence $\mathcal{G}_{k}(M) \simeq \mathcal{G}_{k} \times \prod_{i=1}^{d} \Omega^{2} S U(3)$. Since $\pi_{4}\left(\Omega^{2}(S U(3)) \cong\right.$ $\mathbb{Z} / 3 \mathbb{Z}$ by Lemma 5.1 , we obtain

$$
\pi_{4}\left(\mathcal{G}_{k}(M)\right) \cong \pi_{4}\left(\mathcal{G}_{k}\right) \oplus \bigoplus_{i=1}^{d} \pi_{4}\left(\Omega^{2} S U(3)\right) \cong \pi_{4}\left(\mathcal{G}_{k}\right) \oplus \bigoplus_{i=1}^{d} \mathbb{Z} / 3 \mathbb{Z}
$$

By [S], there is a (3-local) isomorphism $\pi_{4}\left(\mathcal{G}_{k}\right) \cong \mathbb{Z} /(3, k) \mathbb{Z}$. Thus $\pi_{4}\left(\mathcal{G}_{k}(M)\right) \cong$ $\bigoplus_{i=1}^{d} \mathbb{Z} / 3 \mathbb{Z}$ if and only if $(3, k)=1$ and $\pi_{4}\left(\mathcal{G}_{k}(M)\right) \cong \bigoplus_{i=1}^{d+1} \mathbb{Z} / 3 \mathbb{Z}$ if and only if $(3, k)=3$. Consequently, $\pi_{4}\left(\mathcal{G}_{k}(M)\right) \cong \pi_{4}\left(\mathcal{G}_{k^{\prime}}(M)\right)$ if and only if $(3, k)=\left(3, k^{\prime}\right)$.

Combining Lemmas 7.3 and 7.4 we obtain the following.

Proposition 7.5. Let $M$ be a simply-connected 4-manifold. Suppose there is a homotopy equivalence $\mathcal{G}_{k}(M) \simeq \mathcal{G}_{k^{\prime}}(M)$. The following hold:

(a) if $M$ is Spin then $(24, k)=\left(24, k^{\prime}\right)$;

(b) if $M$ is non-Spin then $(12, k)=\left(12, k^{\prime}\right)$.

To conclude, we prove Theorem 1.1. 
Proof of Theorem 1.1. Part (a) is the combination of Corollary 3.2 and Proposition 7.5 (a). Part (b) is the combination of Propositions 6.9 and 7.5 (b).

\section{References}

[AB] M. F. Atiyah and R. Bott, The Yang-Mills equations over Riemann surfaces, Philos. Trans. Roy. Soc. London Ser. A 308 (1983), 523-615. Zbl 0509.14014 MR 0702806

[CS] M. C. Crabb and W. A. Sutherland, Counting homotopy types of gauge groups, Proc. London Math. Soc. 83 (2000), 747-768. Zbl 1024.55005 MR 1781154

[HK1] H. Hamanaka and A. Kono, On $[X, U(n)]$ when $\operatorname{dim} X$ is $2 n$, J. Math. Kyoto Univ. 43 (2003), 333-348. Zbl 1070.55007 MR 2051028

[HK2] Unstable $K^{1}$-group and homotopy type of certain gauge groups, Proc. Roy. Soc. Edinburgh Sect. A 136 (2006), 149-155. Zbl 1103.55004 MR 2217512

[K] A. Kono, A note on the homotopy type of certain gauge groups, Proc. Roy. Soc. Edinburgh Sect. A 117 (1991), 295-297. Zbl 0722.55008 MR 1103296

[KT] A. Kono and S. Tsukuda, A remark on the homotopy type of certain gauge groups, J. Math. Kyoto Univ. 36 (1996), 115-121. Zbl 0865.57018 MR 1381542

[L] G. E. Lang, The evaluation map and EHP sequences, Pacific J. Math. 44 (1973), 201-210. Zbl 0217.20003 MR 0341484

[M] M. Mimura, On the number of multiplications on $S U(3)$ and $S p(2)$, Trans. Amer. Math. Soc. 146 (1969), 473-492. Zbl 0198.56204 MR 0253335

[MT] M. Mimura and H. Toda, Homotopy groups of $S U(3), S U(4)$, and $S p(2)$, J. Math. Kyoto Univ. 3 (1964), 217-250. Zbl 0129.15404 MR 0169242

[S] W. A. Sutherland, Function spaces related to gauge groups, Proc. Roy. Soc. Edinburgh Sect. A 121 (1992), 185-190. Zbl 0761.55007 MR 1169902

[Th1] S. D. Theriault, Odd primary decompositions of gauge groups, Algebr. Geom. Topol. 10 (2010), 535-564. Zbl 1196.55009 MR 2602840

[Th2] , The homotopy types of $S p(2)$-gauge groups, J. Math. Kyoto Univ. 50 (2010), 591-605. Zbl 1202.55004 MR 2723863

[To1] H. Toda, A topological proof of theorems of Bott and Hirzebruch for homotopy groups of unitary groups, Mem. Coll. Sci. Univ. Kyoto Ser. A Math. 32 (1959), 103-119. Zbl 0106.16403 MR 0108790

[To2] Composition methods in homotopy groups of spheres, Ann. of Math. Stud. 49, Princeton Univ. Press, 1962. Zbl 0101.40703 MR 0143217 